\title{
Erratum to: Systematic Review and Meta-Analysis of the Efficacy and Safety of Perampanel in the Treatment of Partial-Onset Epilepsy
}

\author{
Warrington W. Q. Hsu • C. W. Sing •
}

Ying He - Alan J. Worsley • Ian C. K. Wong •

Esther W. Chan

Published online: 14 November 2013

(c) Springer International Publishing Switzerland 2013

Erratum to: CNS Drugs (2013) 27:817-827

DOI 10.1007/s40263-013-0091-9

Page 826, Open Access: The following text should have been included in this section:

"The open access publication fee was funded by Eisai Co., Ltd".

The online version of the original article can be found under doi:10.1007/s40263-013-0091-9.

W. W. Q. Hsu · C. W. Sing - Y. He - A. J. Worsley ·

I. C. K. Wong · E. W. Chan $(\square)$

Department of Pharmacology and Pharmacy, Centre for Safe

Medication Practice and Research, Li Ka Shing Faculty of

Medicine, University of Hong Kong, 2/F Laboratory Block,

21 Sassoon Road, Hong Kong SAR, China

e-mail: ewchan@hku.hk

W. W. Q. Hsu

e-mail: warhsu@hku.hk 